\title{
Tobacco smoking as a risk factor for major depressive disorder: population-based study
}

Julie A. Pasco, Lana J. Williams, Felice N. Jacka, Felicity Ng, Margaret J. Henry, Geoffrey C. Nicholson, Mark A. Kotowicz and Michael Berk

\section{Background}

Smoking is disproportionately prevalent among people with psychiatric illness.

\section{Aims}

To investigate smoking as a risk factor for major depressive disorder.

\section{Method}

A population-based sample of women was studied using case-control and retrospective cohort study designs. Exposure to smoking was self-reported, and major depressive disorder diagnosed using the Structured Clinical Interview for DSM-IV-TR (SCID-I/NP)

\section{Results}

Among 165 people with major depressive disorder and 806 controls, smoking was associated with increased odds for major depressive disorder (age-adjusted odds ratio $(\mathrm{OR})=1.46$, $95 \% \mathrm{Cl}$ 1.03-2.07). Compared with non-smokers, odds for major depressive disorder more than doubled for heavy smokers (>20 cigarettes/day). Among 671 women with no history of major depressive disorder at baseline, 13 of 87 smokers and 38 of 584 non-smokers developed de novo major depressive disorder during a decade of follow-up. Smoking increased major depressive disorder risk by $93 \%$ (hazard ratio $(H R)=1.93,95 \% \mathrm{Cl} 1.02-3.69$ ); this was not explained by physical activity or alcohol consumption.

\section{Conclusions}

Evidence from cross-sectional and longitudinal data suggests that smoking increases the risk of major depressive disorder in women.

\section{Declaration of interest}

J.P., L.W., F.J. and M.B. have received research support from an unrestricted educational grant from Eli Lilly. Funding detailed in Acknowledgements.
Tobacco smoking is a major public health problem and several preventive public health strategies have been implemented. However, smoking remains disproportionately prevalent among people with psychiatric illness ${ }^{1,2}$ and this is often considered within the mental health profession to be a secondary or deferrable treatment goal to the control of the psychiatric illness. It is becoming increasingly clear that smoking is not innocuous to mental health, and may in fact aggravate mental illness or contribute to its onset. On a neurobiological level, this may be related to the impact of nicotine on monoamine neurotransmitter regulation, including dopamine, via the diffuse cholinergic pathways. ${ }^{3}$ This may underlie the circadian dysrhythmicity and hedonic dysregulation in smokers, ${ }^{4}$ and may predispose to the development of mood disorders. Smoking also has other systemic and metabolic consequences that may likewise increase this vulnerability.

There is already evidence that smoking is a risk factor for depression. Association data from cross-sectional studies ${ }^{5,6}$ support evidence from prospective studies to suggest that smoking pre-dates the onset of depression. ${ }^{7,8}$ However, there is only limited longitudinal data in the existing literature, and most longitudinal studies have involved time frames under 2 years, which may not be adequate to demonstrate the insidious effects of nicotine dependence. In this epidemiological study, we investigated the status of tobacco smoking as a risk factor for major depression using not only cross-sectional data but also longitudinal data that extend over a period of 10 years.

\section{Method}

\section{Participants}

This study is nested within the Geelong Osteoporosis Study, a programme of research originally designed to investigate the epidemiology of osteoporosis in Australian women, but recently expanded to examine both psychiatric illness and non-psychiatric diseases. The criterion for inclusion into the Geelong Osteoporosis Study is women listed on the current Australian Commonwealth electoral roll for the region known as the Barwon Statistical Division and the criteria for exclusion are inability to provide informed consent, death, and not able to be contacted. Reasons for non-participation are described elsewhere. ${ }^{9}$ During the period 1994-1997, 1494 women recruited into the Geelong Osteoporosis Study have been prospectively followed for a decade. At the time of writing, a further 208 had been recruited during 2005-2007. A total of 1043 women (aged 20-93 years) participated in a psychiatric assessment during the period 2004-2007, thus fulfilling the inclusion criteria for this study. The Barwon Health Research and Ethics Advisory Committee approved the study, and all participants provided informed, written consent.

\section{Data}

Lifestyle practices including smoking, alcohol consumption, habitual physical activity levels and exposure to disease were self-reported. Smoking was recognised if individuals reported regularly smoking more than one or two cigarettes per day for at least 6 months, and recorded details of smoking included frequency and period of exposure. Alcohol intake was recognised if alcohol was consumed several times per week or every day. Habitual physical activity was classified as active if participants reported 'moving, walking and working energetically and participating in vigorous exercise', otherwise they were classified as sedentary. Cardiovascular disease included hypertension, angina and coronary artery disease; diabetes encompassed both types 1 and 2. Socio-economic status was ascertained using Socio-Economic Index for Areas index scores based on census data from the Australian Bureau of Statistics. ${ }^{10}$ These data were 
used to derive an Index of Economic Resources (IER), which was categorised into five groups, according to quintiles of IER for the study region.

The Structured Clinical Interview for DSM-IV-TR Research Version, Non-patient Edition (SCID-I/NP $)^{11}$ was used to identify women with a lifetime history of major depressive disorder and to determine age at onset. Psychiatric interviews were conducted by trained personnel.

\section{Study designs}

Case-control

Among 1043 women who underwent psychiatric assessment, 237 were diagnosed with major depressive disorder and 806 had no history of major depressive disorder. Exposure to smoking was recognised if smoking was practised prior to the onset of major depression. Sixty-eight individuals were excluded because their age at major depressive disorder onset was less than 20 years (the minimum age for controls) and four were excluded because it was unclear whether smoking pre- or post-dated major depressive disorder onset. Thus, 165 individuals with major depressive disorder and 806 controls were eligible for analysis in this case-control study.

\section{Retrospective cohort}

Among 1043 women who underwent psychiatric assessment, a decade of longitudinal data was available for 835 . Based on retrospective data, 164 were excluded because they had experienced a major depressive disorder episode prior to baseline. Among the 671 women aged 20-84 years with no history of major depressive disorder at baseline and who were thus eligible for analysis in this retrospective cohort study, 51 developed de novo major depressive disorder and 620 remained major depressive disorder-free during follow-up. Participants were classified as smokers if they were current smokers at baseline, otherwise they were classified as non-smokers.

\section{Statistics}

Statistical analyses were performed using Stata (version 9.0) and Minitab (version 13). Standard descriptive statistics were used to characterise the participants in each study.

In the case-control study, participants were selected as cases (people with major depressive disorder) or controls (people with no major depressive disorder), and exposure to smoking was documented for each group. Logistic regression modelling was performed to determine the association between smoking and major depressive disorder. Age was defined as the age at major depressive disorder onset for cases and age at baseline for controls, and was categorised into age groups for analysis. Smoking was investigated as a binary variable and was also categorised into groups according to the average number of cigarettes smoked per day $(0, \leqslant 10,11-20,>20$ cigarettes/day). Age, socio-economic status, physical illness, physical activity and alcohol consumption were tested in the models as potential confounders and effect modifiers.

In the cohort study, participants with no history of major depressive disorder at baseline were selected, categorised as current smokers or not, and followed until a first major depressive disorder episode or until the end of the follow-up period. The effect of smoking on development of de novo major depressive disorder was examined using multivariate Cox proportional hazards regression analysis, using age as the time axis. The proportional hazards assumptions were checked using
Schoenfeld residuals before and after adjusting for potential confounding by socio-economic status, physical illness, physical activity and alcohol consumption.

\section{Results}

\section{Case-control study}

Characteristics of the participants involved in the case-control analysis are shown in Table 1. Participants with major depressive disorder were younger and were more often smokers. Exposure to smoking was documented for 73 of the 165 people with major depressive disorder and for 269 of 806 controls. Prevalence of smoking was thus greater among women with major depressive disorder (0.44 (95\% CI 0.37-0.52) v. 0.33 (95\% CI 0.30-0.37), $P=0.008$ ). Exposure to smoking increased the odds for major depressive disorder (odds ratio $\mathrm{OR}=1.58,95 \%$ CI 1.13-2.23, $P=0.008)$ and this association persisted, albeit attenuated, after adjusting for age (age-adjusted $\mathrm{OR}=1.46,95 \%$ CI 1.03-2.07, $P=0.031$ ). Socio-economic status did not confound the association between smoking and major depressive disorder (age- and socio-economic status-adjusted $\mathrm{OR}=1.49$, 95\% CI 1.05-2.11, $P=0.026)$. Similarly, the association was not explained by a history of self-reported cardiovascular disease or diabetes (adjusted $\mathrm{OR}=1.47,95 \%$ CI $1.04-2.09, P=0.030$ ). Among the 342 smokers, participants with major depressive disorder smoked more heavily than those in the control group (median (interquartile range), 15 (10-20) v. 10 (8-20) cigarettes per day, $P=0.059)$. Compared with non-smokers, the odds for major depressive disorder tended to increase 1.47-fold for women who smoked 11-20 cigarettes per day $(P=0.094)$ and more than doubled for those who smoked more than 20 cigarettes per day $(P=0.003)$ (Table 2).

Being physically active was found to be protective against major depressive disorder (age-adjusted $\mathrm{OR}=0.58$, 95\% CI $0.37-$ $0.91, P=0.017)$. None the less, the association between smoking and major depressive disorder was not explained by differences in physical activity. The independent relationships between smoking and physical activity on the risk for major depressive disorder are shown in Fig. 1. Alcohol consumption did not affect this association.

\section{Retrospective cohort study}

Characteristics of women included in this analysis are shown in Table 3. Among 87 women who were current smokers at baseline, 13 developed de novo major depressive disorder during 781 person-years of observation, whereas among 584 non-smokers, 38 developed major depressive disorder during 5384 person-years of observation. Estimated rates of major depressive disorder were 16.6 (95\% CI 9.7-28.7) per 1000 person-years for smokers and 7.1 (95\% CI 5.1-9.7) per 1000 person-years for non-smokers.

Exposure to smoking was found to increase the risk for developing a first episode of major depressive disorder by $93 \%$, hazard ratio $(\mathrm{HR})=1.93 \quad(95 \%$ CI $1.02-3.69, \quad P=0.045)$. A Kaplan-Meier survival plot showing the probability of remaining free of major depressive disorder over a 10-year period for women exposed and unexposed to smoking at baseline is shown in Fig. 2. Adjustment for socio-economic status enhanced the risk (adjusted $\mathrm{HR}=2.01,95 \%$ CI 1.03-3.93, $P=0.042$ ). Further adjustment for alcohol consumption, physical activity or physical illness did not attenuate this association.

\section{Discussion}

This study provides both cross-sectional and longitudinal evidence consistent with the hypothesis that tobacco smoking is 


\begin{tabular}{|c|c|c|c|}
\hline & $\begin{array}{l}\text { Major depressive disorder } \\
\qquad(n=165)\end{array}$ & $\begin{array}{l}\text { No major depressive disorder } \\
\qquad(n=806)\end{array}$ & $P$ \\
\hline Age, years: median (IQR) & $33(26-45)$ & $43(29-59)$ & $<0.0001$ \\
\hline Smokers, $n(\%)$ & $73(44)$ & $269(33)$ & 0.008 \\
\hline Socio-economic status, $n$ (\%) & & & 0.857 \\
\hline Quintile 1 (low) & $23(14)$ & $125(16)$ & \\
\hline Quintile 2 & $33(20)$ & $184(23)$ & \\
\hline Quintile 3 & $41(25)$ & $183(23)$ & \\
\hline Quintile 4 & $29(18)$ & $142(18)$ & \\
\hline Quintile 5 & $39(24)$ & $172(21)$ & \\
\hline Alcohol users, $n(\%)$ & $28(17)$ & $144(18)$ & 0.784 \\
\hline Physically active, $n$ (\%) & 31 (19) & $168(21)$ & 0.551 \\
\hline Cardiovascular disease, $n$ (\%) & $56(34)$ & $240(30)$ & 0.290 \\
\hline Diabetes, $n$ (\%) & $11(7)$ & $48(6)$ & 0.727 \\
\hline
\end{tabular}

\begin{tabular}{|lcc|}
\hline $\begin{array}{l}\text { Table } 2 \\
\text { Cigarettes per day }\end{array}$ & Unadjusted $\mathrm{OR}(95 \% \mathrm{Cl})$ & Age-adjusted OR (95\% Cl) \\
\hline$<10$ & $1.02(0.56-1.88)$ & $0.88(0.48-1.64)$ \\
\hline $11-20$ & $1.62(1.04-2.52)$ & $1.47(0.94-2.32)$ \\
\hline$>20$ & $2.17(1.31-3.58)$ & $2.18(1.31-3.65)$ \\
\hline
\end{tabular}

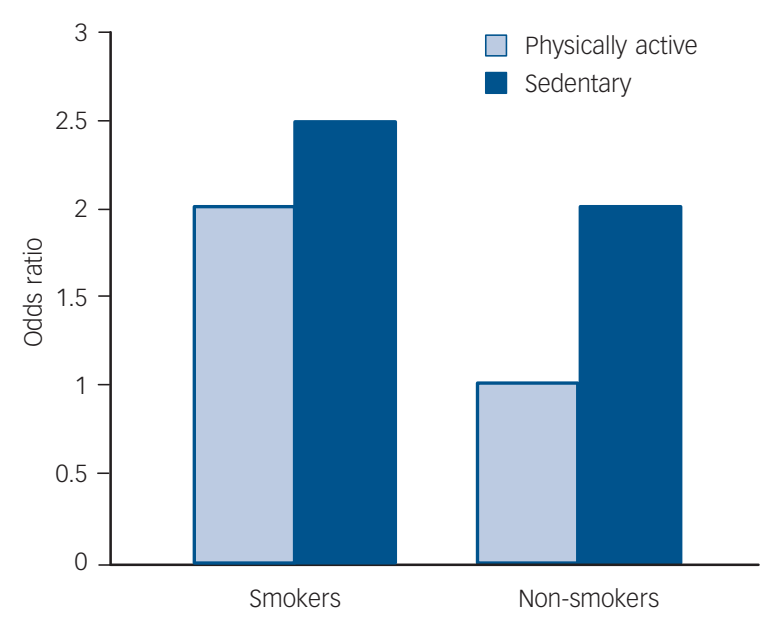

Fig. 1 Independent contributions of tobacco smoking and physical activity to the risk for major depression. Non-smokers who are physically active form the reference group.

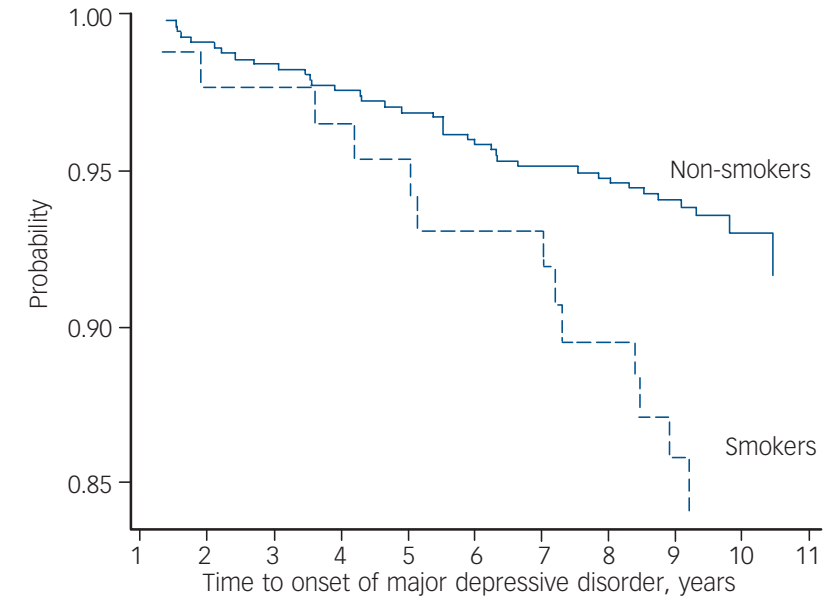

Fig. 2 Survival plot (Kaplan-Meier) showing the probability of remaining free of de novo major depressive disorder over a 10-year period for smokers and non-smokers at baseline. associated with major depression. Our cross-sectional data demonstrate that exposure to smoking is associated with a 1.46fold increase in the odds for major depressive disorder. Furthermore, our findings are suggestive of a dose-dependent association, with more than a two-fold increase in the odds of major depressive disorder for heavy smokers compared with non-smokers. With the advantage of temporal sequencing, our longitudinal data demonstrate that smoking is associated with a near doubling of risk for developing de novo major depressive disorder over a 10year period. These effects were independent of age and physical activity, and not explained by alcohol consumption.

Other cross-sectional studies have reported increased odds of depression in smokers, ${ }^{5,6}$ with results retaining statistical significance after adjusting for other major risk factors. ${ }^{6}$ Prospective studies, although limited, have further strengthened the suspected role of smoking in depression. In an 11-year population-based longitudinal Norwegian study, the hazard ratio for a first depressive episode increased with smoking in a dosedependent fashion, such that the heaviest smokers (exceeding 20 cigarettes per day) had over four times the risk of those who had never smoked. ${ }^{7}$ Increased incidence of major depression in smokers has been reported in other shorter studies, ${ }^{12,13}$ including data from adolescents. ${ }^{14}$ Longitudinal studies have also shown a reverse relationship, in which the presence of depression increased the risk of smoking progression. ${ }^{12,13}$ Positive effects on psychomotor performance and enhanced craving, as demonstrated in a 


\begin{tabular}{|c|c|c|c|}
\hline & Smokers $(n=87)$ & Non-smokers $(n=584)$ & $P$ \\
\hline Age, years: median (IQR) & $39(31-52)$ & $50(37-63)$ & $<0.0001$ \\
\hline Major depressive disorder, $n$ (\%) & $13(15)$ & $38(7)$ & 0.006 \\
\hline Socio-economic status, $n$ (\%) & & & 0.001 \\
\hline Quintile 1 (low) & $26(30)$ & $93(16)$ & \\
\hline Quintile 2 & $19(22)$ & $137(23)$ & \\
\hline Quintile 3 & $22(25)$ & $107(18)$ & \\
\hline Quintile 4 & $6(7)$ & $114(20)$ & \\
\hline Quintile 5 & $14(16)$ & $133(23)$ & \\
\hline Alcohol users, $n(\%)$ & $20(23)$ & $96(16)$ & 0.132 \\
\hline Physically active, $n$ (\%) & $14(16)$ & $69(12)$ & 0.258 \\
\hline Cardiovascular disease, $n(\%)$ & $20(23)$ & $220(38)$ & 0.006 \\
\hline Diabetes, $n$ (\%) & $3(3)$ & $44(8)$ & 0.129 \\
\hline
\end{tabular}

physiological study, ${ }^{15}$ may be pertinent factors for this observation. Other studies have provided support for a third possibility, that depression and smoking coexist as epiphenomena of a common underlying cause, such as genetic factors. ${ }^{16,17}$ The efficacy of bupropion in the treatment of both depression and nicotine dependence ${ }^{18}$ may indicate some commonality between the two conditions on a neurochemical level.

Dopamine is one such factor, which is believed to have a dual role in depression and in the mechanism of addiction. Neurochemical studies of depression, particularly with psychomotor retardation, reported an association with diminished dopamine metabolism, as evidenced by decreased levels of cerebrospinal fluid homovanillic acid. ${ }^{19,20}$ Reduced striatal dopamine function has also been shown in dopamine $\mathrm{D}_{2}$ receptor neuroimaging binding studies. ${ }^{21,22}$ Dopamine is regarded as the central neurotransmitter of reward, and as having a key role in the reinforcement of the pathways to addiction. ${ }^{23}$ Dysregulation of the dopaminergic system in addictive states is also a plausible mechanistic pathway to depressive vulnerability. ${ }^{24}$

Smoking-induced oxidative stress is another factor. Tobacco smoke generates free radicals, causing lipid peroxidation, oxidation of proteins and other tissue damage in smokers. ${ }^{25,26}$ Depression has been characterised by elevated markers of oxidative stress ${ }^{27-29}$ that demonstrates a positive correlation with depressive severity ${ }^{30}$ and a return to normal levels after treatment. ${ }^{28,29}$ It seems plausible that depression could be among the oxidative stress sequelae of smoking.

There are several strengths and potential weaknesses in our study. The length of the follow-up period is a key strength, especially when published longitudinal studies have rarely exceeded a few years at most. Given that smoking effects insidious biochemical changes that are naturally accommodated by the body's homeostatic responses, long-term sequelae such as depression, cancers, cardiovascular and pulmonary diseases may only be reliably demonstrated over an extended time frame. Recall limitations may have affected our ability to accurately diagnose the time of onset of depressive episodes and, in the case-control analysis, the potential exists for differential recall bias of smoking practices. However, as this study was nested within a larger prospective study, the latter risk was minimised as exposure to smoking had been documented prior to psychiatric interview. Furthermore, documentation of exposure to smoking and assessment of outcome were performed by different study personnel. The duration of smoking prior to the onset of depression was unknown, precluding estimation of duration of exposure on depression. Inconsistencies in the number of cigarettes smoked per day may have resulted in misclassification of smoking frequency in the case-control analysis but the apparent dose-dependent association strengthens the notion that smoking is a risk factor for major depression. Small numbers limited a comparable investigation in the longitudinal analysis. Also in the longitudinal analysis, changes in exposure status during follow-up have not been identified. Finally, as in all observational studies, there may be unrecognised confounding. We relied on self-reported history of cardiovascular disease and diabetes as indicators of physical illness that may be affected by smoking status. However, we cannot exclude possible confounding by unrecognised comorbidity as individuals were not clinically screened for all potential physical illnesses. Physical activity and alcohol consumption were explored as concomitant lifestyle factors with a potential for confounding because physical activity had been previously reported as protective against depression, ${ }^{31}$ whereas physical inactivity ${ }^{32-34}$ and alcohol misuse ${ }^{6}$ are regarded as risk factors. In this study, alcohol consumption did not appear to confound the association between smoking and depression; however, we acknowledge that our criteria for alcohol consumption may have been too broad to confidently exclude its contribution. Other factors predisposing to depression, such as personality traits, developmental and family history of depression, IQ or stress, were not considered as these data were not available.

Within these limitations, however, our data corroborate literature that reveals a malevolent role of smoking in depression and suggest that greater efforts are required in targeting smoking as a routine intervention. ${ }^{35}$ Depression's status as a leading cause of global disease burden, ${ }^{36,37}$ one that is not anticipated to yield in the coming decades, ${ }^{36}$ can only underscore the potential impact of any effective preventive measures.

Julie A. Pasco, BSc(Hons), Dip Ed, PhD, MEpi, Lana J. Williams, BPsych, Grad Dip App Psych, PhD, Felice N. Jacka, BA, PgDipSc, Felicity Ng, MBBS, FRANZCP, Margaret J. Henry, BSC(Hons), PhD, Geoffrey C. Nicholson, MBBS, PhD, FRACP, FRCP, Mark A. Kotowicz, MBBS, FRACP, Department of Clinical and Biomedical Science: Barwon Health, The University of Melbourne, Victoria; Michael Berk, MBBch, MMed, FF(Psych)SA, FRANZCP, PhD, Department of Clinical and Biomedical Science: Barwon Health, The University of Melbourne, Victoria, and Orygen Research Centre and Mental Health Research Institute, Australia.

Correspondence: Julie A. Pasco, Epidemiology and Biostatistics Unit, Department of Clinical and Biomedical Sciences: Barwon Health, The University of Melbourne, PO Box 281, Geelong 3220, Australia. Email:

juliep@barwonhealth.org.au

First received 25 Oct 2007, final revision 11 Apr 2008, accepted 16 May 2008

\section{Acknowledgements}

The study was funded by the National Health and Medical Research Council of Australia, supported by an unrestricted educational grant from Eli Lilly. Postgraduate scholarships 
were provided by the University of Melbourne, Faculty of Medicine, Dentistry and Health Sciences and the Australian Rotary Health Research Fund. We thank Sharon Brennan for obtaining the socio-economic data for the study.

\section{References}

1 Grant BF, Hasin DS, Chou SP, Stinson FS, Dawson DA. Nicotine dependence and psychiatric disorders in the United States: results from the national epidemiologic survey on alcohol and related conditions. Arch Gen Psychiatry 2004; 61: 1107-15

2 Kilian R, Becker T, Kruger K, Schmid S, Frasch K. Health behavior in psychiatric in-patients compared with a German general population sample. Acta Psychiatr Scand 2006; 114: 242-8.

3 Dani JA, De Biasi M. Cellular mechanisms of nicotine addiction. Pharmacol Biochem Behav 2001; 70: 439-46.

4 Adan A, Prat G, Sanchez-Turet M. Effects of nicotine dependence on diurnal variations of subjective activation and mood. Addiction 2004; 99: 1599-607.

5 Harlow BL, Cohen LS, Otto MW, Spiegelman D, Cramer DW. Prevalence and predictors of depressive symptoms in older premenopausal women: the Harvard Study of Moods and Cycles. Arch Gen Psychiatry 1999; 56: 418-24.

6 Hamalainen J, Kaprio J, Isometsa E, Heikkinen M, Poikolainen K, Lindeman S, Aro $\mathrm{H}$. Cigarette smoking, alcohol intoxication and major depressive episode in a representative population sample. J Epidemiol Community Health 2001 55: $573-6$

7 Klungsoyr O, Nygard JF, Sorensen T, Sandanger I. Cigarette smoking and incidence of first depressive episode: an 11-year, population-based follow-up study. Am J Epidemiol 2006; 163: 421-32.

8 Steuber TL, Danner F. Adolescent smoking and depression: which comes first? Addict Behav 2006; 31: 133-6.

9 Henry MJ, Pasco JA, Nicholson GC, Seeman E, Kotowicz MA. Prevalence of osteoporosis in Australian women: Geelong Osteoporosis Study. J Clin Densitom 2000; 3: 261-8.

10 Adhikari P. Socio-economic indexes for areas: introduction, use and plans for 2006. Presented at the Census Analysis Conference, 18-19 July 2006. Australian Bureau of Statistics.

11 First MB, Spitzer RL, Gibbon M, Williams, JBW. Structured Clinical Interview for DSM-IV-TR Axis I Disorders, Research Version (Non-patient edn) (SCID-I/ NP). Biometrics Research, New York State Psychiatric Institute, 2002.

12 Breslau N, Kilbey MM, Andreski P. Nicotine dependence and major depression. New evidence from a prospective investigation. Arch Gen Psychiatry 1993; 50: 31-5.

13 Breslau N, Peterson EL, Schultz LR, Chilcoat HD, Andreski P. Majo depression and stages of smoking. A longitudinal investigation. Arch Gen Psychiatry 1998; 55: 161-6.

14 Brown RA, Lewinsohn PM, Seeley JR, Wagner EF. Cigarette smoking, major depression, and other psychiatric disorders among adolescents. J Am Acad Child Adolesc Psychiatry 1996; 35: 1602-10.

15 Malpass D, Higgs S. Acute psychomotor, subjective and physiological responses to smoking in depressed outpatient smokers and matched controls. Psychopharmacology (Berl) 2007; 190: 363-72.

16 Kendler KS, Neale MC, MacLean CJ, Heath AC, Eaves LJ, Kessler RC. Smoking and major depression. A causal analysis. Arch Gen Psychiatry 1993; 50: 36-43.

17 Korhonen T, Broms U, Varjonen J, Romanov K, Koskenvuo M, Kinnunen T, Kaprio J. Smoking behaviour as a predictor of depression among Finnish men and women: a prospective cohort study of adult twins. Psychol Med 2007; 37: 705-15.
18 Foley KF, DeSanty KP, Kast RE. Bupropion: pharmacology and therapeutic applications. Expert Rev Neurother 2006; 6: 1249-65.

19 Korf J, van Praag HM. Retarded depression and the dopamine metabolism Psychopharmacologia 1971; 19: 199-203.

20 Post RM, Kotin J, Goodwin FK, Gordon EK. Psychomotor activity and cerebrospinal fluid amine metabolites in affective illness. Am J Psychiatry 1973; 130: 67-72.

21 Ebert $D$, Feistel $H$, Loew $T$, Pirner A. Dopamine and depression-striatal dopamine D2 receptor SPECT before and after antidepressant therapy. Psychopharmacology (Berl) 1996; 126: 91-4.

22 Martinot M, Bragulat V, Artiges $E$, Dolle F, Hinnen F, Jouvent R, Martinot J. Decreased presynaptic dopamine function in the left caudate of depressed patients with affective flattening and psychomotor retardation. Am J Psychiatry 2001; 158: 314-6.

23 Di Chiara G, Bassareo V. Reward system and addiction: what dopamine does and doesn't do. Curr Opin Pharmacol 2007; 7: 69-76.

24 Malhi GS, Berk M. Does dopamine dysfunction drive depression? Acta Psychiatr Scand Suppl 2007; 433: 116-24

25 Hulea SA, Olinescu R, Nita S, Crocnan D, Kummerow FA. Cigarette smoking causes biochemical changes in blood that are suggestive of oxidative stress: a case-control study. J Environ Pathol Toxicol Oncol 1995; 14: 173-80.

26 Ozguner F, Koyu A, Cesur G. Active smoking causes oxidative stress and decreases blood melatonin levels. Toxicol Ind Health 2005; 21: 21-6.

27 Ozcan ME, Gulec M, Ozerol E, Polat R, Akyol O. Antioxidant enzyme activities and oxidative stress in affective disorders. Int Clin Psychopharmacol 2004; 19: 89-95.

28 Bilici M, Efe H, Koroglu MA, Uydu HA, Bekaroglu M, Deger O. Antioxidative enzyme activities and lipid peroxidation in major depression: alterations by antidepressant treatments. J Affect Disord 2001; 64: 43-51.

29 Khanzode SD, Dakhale GN, Khanzode SS, Saoji A, Palasodkar R. Oxidative damage and major depression: the potential antioxidant action of selective serotonin re-uptake inhibitors. Redox Rep 2003; 8: 365-70.

30 Yanik M, Erel O, Katoi M. The relationship between potency of oxidative stress and severity of depression. Acta Neuropsychiatrica 2004; 16: 200-3.

31 Strawbridge WJ, Deleger S, Roberts RE, Kaplan GA. Physical activity reduces the risk of subsequent depression for older adults. Am J Epidemiol 2002; 156: 328-34.

32 Brown WJ, Ford JH, Burton NW, Marshall AL, Dobson AJ. Prospective study of physical activity and depressive symptoms in middle-aged women. Am J Prev Med 2005; 29: 265-72.

33 Camacho TC, Roberts RE, Lazarus NB, Kaplan GA, Cohen RD. Physical activity and depression: evidence from the Alameda County Study. Am J Epidemiol 1991; 134: 220-31.

34 Farmer ME, Locke BZ, Moscicki EK, Dannenberg AL, Larson DB, Radloff LS Physical activity and depressive symptoms: the NHANES I Epidemiologic Follow-up Study. Am J Epidemiol 1988; 128: 1340-51.

35 Berk M. Should we be targeting smoking as an intervention? Acta Neuropsychiatrica 2007; 19: 131-2.

36 Murray CJ, Lopez AD. Alternative projections of mortality and disability by cause 1990-2020: Global Burden of Disease Study. Lancet 1997; 349: 1498-504.

37 Mathers CD, Vos ET, Stevenson CE, Begg SJ. The Australian Burden of Disease Study: measuring the loss of health from diseases, injuries and risk factors. Med J Aust 2000; 172: 592-6. 